\title{
Reconstruction of $f(T)$ gravity from the Holographic dark energy
}

\author{
Surajit Chattopadhyay ${ }^{1, *}$ and Antonio Pasqua ${ }^{2,+}$ \\ ${ }^{1}$ Pailan College of Management and Technology, Bengal Pailan Park, Kolkata, India. \\ ${ }^{2}$ Department of Physics, University of Trieste, Trieste, Italy.
}

(Dated: November 5, 2018)

\begin{abstract}
Among different candidates to play the role of Dark Energy (DE), modified gravity has emerged as offering a possible unification of Dark Matter (DM) and DE. The purpose of this work is to develop a reconstruction scheme for the modified gravity with $f(T)$ action using holographic energy density. In the framework of the said modified gravity we have considered the equation of state of the Holographic DE (HDE) density. Subsequently we have developed a reconstruction scheme for modified gravity with $f(T)$ action. Finally we have obtained a modified gravity action consistent with the HDE scenario.
\end{abstract}

PACS numbers: 98.80.-k, 95.36.+x, 04.50.Kd

\section{INTRODUCTION}

Cosmological observations obtained with Supernovae Ia (SNeIa), the Cosmic Microwave Background (CMB) radiation anisotropies, the Large Scale Structure (LSS) and X-ray experiments have well established the accelerated expansion of our universe [1 7]. A missing energy component also known as Dark Energy (DE) with negative pressure is widely considered by scientists as responsible of this accelerated expansion. Recent analysis of cosmological observations indicates that the two-thirds of the total energy of the universe is been occupied by the DE whereas DM occupies almost the remaining part (the baryonic matter represents only a few percent of the total energy density of the universe) [8]. The contribution of the radiation is practically negligible.

The nature of DE is still unknown and many candidates have been proposed in order to describe it [9-16]. The simplest one is represented by a tiny positive cosmological constant, with a negative constant equation of state $(\mathrm{EoS})$ parameter $\omega$, i.e. $\omega=-1$. However, cosmologists know that the cosmological constant suffers from two well-known difficulties, the fine-tuning and the cosmic coincidence problems: the former asks why the vacuum energy density is so small (of the order of

*Electronic address: surajit2008@yahoo.co.in, surajcha@iucaa.ernet.in

${ }^{\dagger}$ Electronic address: toto.pasqua@gmail.com 
$10^{-123}$ smaller than what we observe) and the latter says why vacuum energy and DM are nearly equal today [17, 18].

As possible alternative to cosmological constant, dynamical scalar field models have been proposed some of which are quintessence [19 22], phantom [23, 24], f-essence [25, 26] and K-essence [27 29]. An important advance in the studies of black hole theory and string theory is the suggestion of the so called holographic principle which was proposed by Fischler and Susskind in 1998 [30]. According to the holographic principle, the number of degrees of freedom of a physical system should be finite and should scale with its bounding area rather than with its volume [31] and it should be constrained by an infrared cut-off [32]. The Holographic DE (HDE), based on the holographic principle, is one of the most studied models of DE [33 45]. The HDE models have also been constrained and tested by various astronomical observations [46, 47].

Applying the holographic principle to cosmology, the upper bound of the entropy contained in the universe can be obtained. Following this line, Li [48] suggested as constraint on the energy density of the universe $\rho_{\Lambda} \leq 3 \gamma M_{p}^{2} L^{-2}$, where $\gamma$ is a numerical constant, $L$ is the IR cut-off radius and $M_{p}$ is the reduced Planck mass. The equality sign holds when the holographic bound is saturated. Importance of modified gravity for late acceleration of the universe has been reviewed [49, 50]. Various modified gravity theories have been proposed so far: some of the most studied include $f(R)$ [51], $f(G)$ [52, 53], Horava-Lifshitz [54] and Gauss-Bonnet [55] theories. Recently, a new theory of gravity known as $f(T)$ gravity, which is formulated in a space-time possessing absolute parallelism [56, 57], has been proposed.

Fundamental aspects of $f(T)$ gravity have been recently studied [58, 59]. In the $f(T)$ theory of gravity, the teleparallel Lagrangian density described by the torsion scalar $T$ has been promoted to be a function of $T$, i.e. $f(T)$, in order to account for the late time cosmic acceleration [60]. In a recent work, Jamil et al [61] examined the interacting DE model in $f(T)$ cosmology assuming $\mathrm{DE}$ as a perfect fluid and choosing a specific cosmologically viable form $f(T)=\beta \sqrt{T}$. Statefinder diagnostic of $f(T)$ gravity has been studied in [62]. Purpose of the present work is to develop a reconstruction scheme for the modified gravity with $f(T)$ action using holographic energy density. 


\section{RECONSTRUCTION OF $f(T)$ GRAVITY}

In the framework of $f(T)$ theory, the action of modified teleparallel action is given by [63]:

$$
I=\frac{1}{16 \pi G} \int d^{4} x \sqrt{-g}\left[f(T)+L_{m}\right]
$$

where $L_{m}$ is the Lagrangian density of the matter inside the universe, $G$ is the gravitational constant and $g$ is the determinant of the metric tensor $g^{\mu \nu}$. We consider a flat Friedmann-Robertson-Walker (FRW) universe filled with the pressureless matter. Choosing $(8 \pi G=1)$, the modified Friedman equations in the framework of $f(T)$ gravity are given by [63, 64]:

$$
\begin{aligned}
H^{2} & =\frac{1}{3}\left(\rho+\rho_{T}\right), \\
2 \dot{H}+3 H^{2} & =-\left(p+p_{T}\right),
\end{aligned}
$$

where

$$
\begin{aligned}
\rho_{T} & =\frac{1}{2}\left(2 T f_{T}-f-T\right), \\
p_{T} & =-\frac{1}{2}\left[-8 \dot{H} T f_{T T}+(2 T-4 \dot{H}) f_{T}-f+4 \dot{H}-T\right],
\end{aligned}
$$

and [64]:

$$
T=-6\left(H^{2}\right)
$$

Earlier, Setare [65] reconstructed $f(R)$ gravity from HDE. In this Section, we shall discuss a reconstruction of $f(T)$ gravity in HDE scenario. We must emphasize that reconstruction of $f(T)$ gravity has been already studied in [66] and a general DE review which discusses the reconstruction in $f(T)$ gravity can be found in [67].

Following [65], the HDE density is chosen as:

$$
\rho_{\Lambda}=\frac{3 c^{2}}{R_{h}^{2}},
$$

where $R_{h}$ represents the future event horizon and $c$ is a constant.

The expression of $R_{h}$ is given by:

$$
R_{h}=a \int_{t}^{\infty} \frac{d t}{a}=a \int_{a}^{\infty} \frac{d a}{H a^{2}}
$$

The dimensionless DE is defined by using the critical energy density $\rho_{c r}=3 H^{2}$ as follow:

$$
\Omega_{\Lambda}=\frac{\rho_{\Lambda}}{\rho_{c r}}=\frac{c^{2}}{R_{h}^{2} H^{2}} .
$$


The time derivative of the future horizon is given by:

$$
\dot{R}_{h}=R_{h} H-1=\frac{c}{\sqrt{\Omega_{\Lambda}}}-1
$$

Using the conservation equation, the EoS parameter for HDE has been obtained by [65] as follow:

$$
\omega_{\Lambda}=-\left(\frac{1}{3}+\frac{2 \sqrt{\Omega_{\Lambda}}}{3 c}\right)
$$

In order to reconstruct $f(T)$ gravity in HDE scenario, we replace the energy density of Eq. (2) with $\rho_{\Lambda}$ and hence we get:

$$
\rho_{\Lambda}=6 H^{2} f_{T}+\frac{1}{2} f(T)
$$

Using Eq. (9) in Eq. (12), we can write:

$$
6 H^{2} \Omega_{\Lambda}=12 H^{2} f_{T}+f(T)
$$

Hence, we get

$$
f(T)=6 H^{2} \Omega_{\Lambda}-12 H^{2} f_{T}=-T\left(\Omega_{\Lambda}-2 f_{T}\right) .
$$

We now consider Eq. (3), where $p$ would be replaced by $p_{\Lambda}$. As $\omega_{\Lambda}=p_{\Lambda} / \rho_{\Lambda}$, we can write

$$
p_{\Lambda}=3 H^{2} w_{\Lambda} \Omega_{\Lambda}
$$

where $\omega_{\Lambda}$ is given in Eq. (111). Using Eqs. (3), (5) and (15), we get:

$$
3 \omega_{\Lambda} H^{2} \Omega_{\Lambda}=-4 \dot{H} T f_{T T}+(T-2 \dot{H}) f_{T}-\frac{1}{2} f(T) .
$$

Subsequently, using $T=-6 H^{2}$, we can write:

$$
3 \omega_{\Lambda} \Omega_{\Lambda}=24 \dot{H} f_{T T}-\left(6+\frac{2 \dot{H}}{H^{2}}\right) f_{T}-\frac{1}{2 H^{2}} f(T) .
$$

For scale factor $a(t)$ we shall consider the solution [65]:

$$
a(t)=a_{0}\left(t_{s}-t\right)^{n}
$$

where, $a_{0}, t_{s}$ and $n$ are constants. Hence:

$$
\begin{aligned}
H & =-\frac{n}{t_{s}-t} \\
T & =-\frac{6 n^{2}}{\left(t_{s}-t\right)^{2}}, \\
\Omega_{\Lambda} & =c^{2}\left(1-\frac{1}{n}\right)^{2} .
\end{aligned}
$$


The solution of Eq. (14) is given by:

$$
f(T)=\left[\frac{c(n-1)}{n}\right]^{2}(T-2)+C_{1} e^{-\frac{T}{2}},
$$

where $C_{1}$ is a constant.

Instead, the solution of Eq. (17) is given by:

$$
f(T)=\frac{\alpha}{\beta-3} T+T^{\frac{\beta_{2}-\beta_{1}-\sqrt{\left(\beta_{1}-\beta_{2}\right)^{2}+12 \beta_{2}}}{2 \beta_{2}}}\left(T^{\frac{\sqrt{\left(\beta_{1}-\beta_{2}\right)^{2}+12 \beta_{2}}}{\beta_{2}}} C_{2}+C_{3}\right),
$$

where

$$
\begin{aligned}
\alpha & =\frac{c^{2}(n-1)^{2}(2-3 n)}{n^{3}}, \\
\beta_{1} & =-6+\frac{2}{n} \\
\beta_{2} & =\frac{4}{n}
\end{aligned}
$$

and $C_{2}$ and $C_{3}$ are two constants.

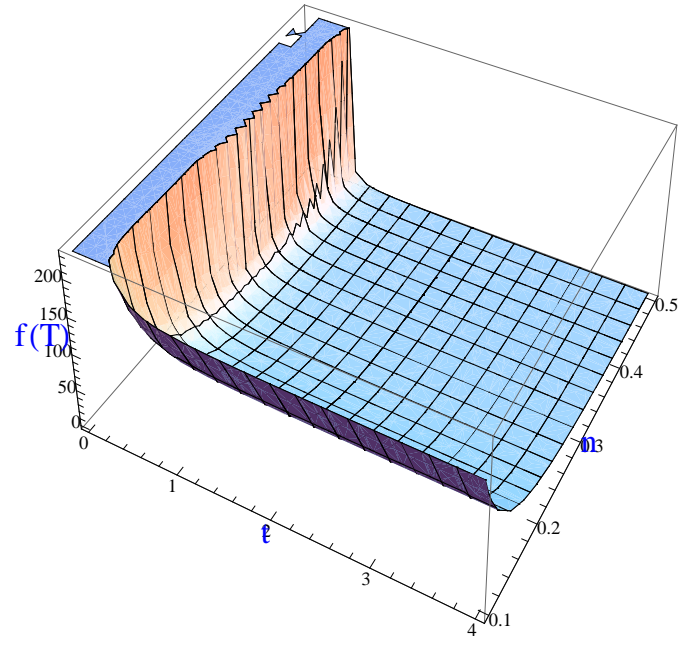

FIG. 1: This Figure plots evolution of reconstructed $f(T)$ for solution given in Eq. (22) and we find $f(T) \geq 0$ with the evolution of the universe for a range of $n$. The $x$-axis plots $t$ and $y$-axis plots $n$.

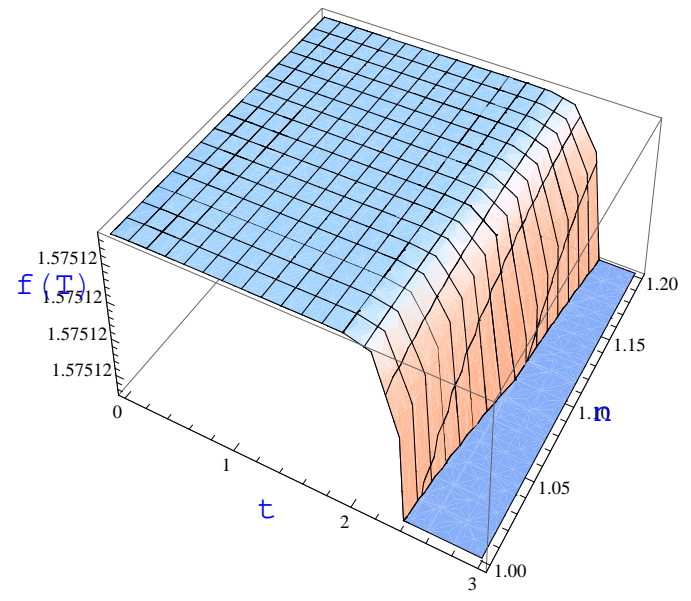

FIG. 2: This Figure plots evolution of reconstructed $f(T)$ for solution given in Eq. (23) and we find $f(T) \geq 0$ with the evolution of the universe for a range of $n$. The $x$-axis plots $t$ and $y$-axis plots $n$.

The solutions for $f(T)$ are now studied graphically. In Figure 1, we plot the solution $f(T)$ given in Eq. (22) against $t$ and $n$. The three dimensional plot shows a decaying behavior of $f(T)$ with evolution of the universe. Furthermore, the rate of decay is increasing with increase in the value of $n$. Similarly, when we plot the expression of $f(T)$ given in (23) in Figure 2, we find a 


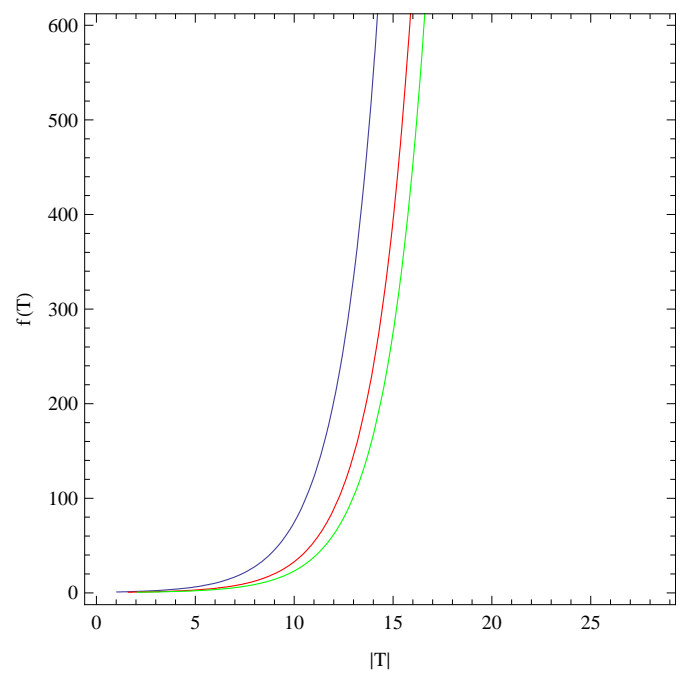

FIG. 3: This Figure plots $f(T)$ for solution given in Eq. (22) against $|T|$ for different values of $n$.

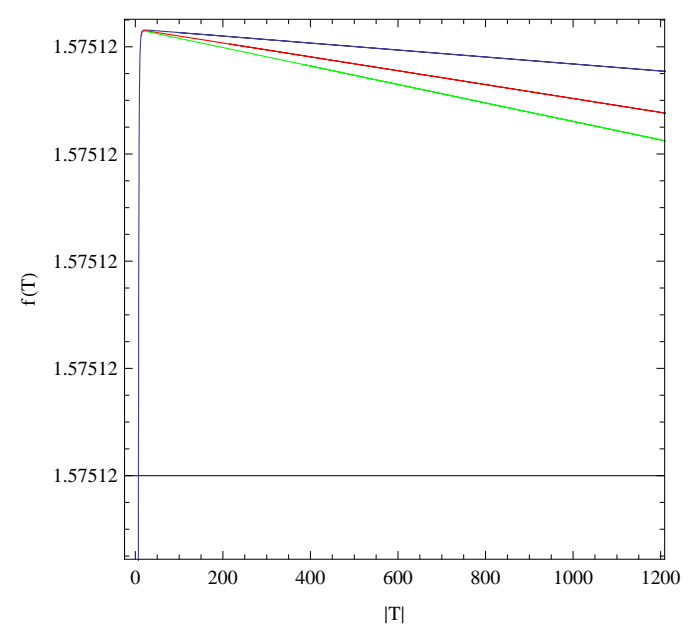

FIG. 4: This Figure plots $f(T)$ for solution given in Eq. (23) against $|T|$ for different values of $n$.

decaying pattern of $f(T)$ with passage of cosmic time. However, contrary to what happened in the earlier case, the rate of decay is less in the case of higher values of $n$. In Figures 3 and 4 we have viewed the behavior of $f(T)$ with variation in $T$. We observe that for the solution corresponding to Eq. (22), the $f(T)$ increases with increase in absolute value of $T$. On the other hand, the $f(T)$ obtained from Eq. (223) decays with $|T|$. It has been discussed in reference [68] that satisfaction of the above condition is a sufficient condition for a realistic model. From Figure 3, we understand that $f(T) \rightarrow 0$ as $T \rightarrow 0$ for the solution obtained from Eq. (22). However, this does not occur for the solution obtained from Eq. (23). Thus, it may be stated that the solution obtained in Eq. (22) is a more realistic model than that obtained in Eq. (23).

\section{CONCLUSION}

Among different candidates to play the role of the DE, modified gravity has emerged as a possible unification of DM and DE. The present work aims at a cosmological application of the HDE density in the framework of a modified gravity, named as $f(T)$ gravity. In the framework of the said modified gravity, we have considered the equation of state of the HDE density. Subsequently, we have developed a reconstruction scheme for modified gravity with $f(T)$ action. Considering De density given in Eq. (4) in holographic form and then by assuming a simple solution for the scale factor $a(t)$ as given in Eq. (18), we have obtained a solution for differential 
equation for $f(T)$ in Eq. (22). Again, by considering the DE pressure in holographic form, we have created a differential equation for $f(T)$ in Eq. (17) and using the same simple solution for the scale factor, we derived $f(T)$. It has been revealed that the solution of Eq. (22) seems to be more realistic than that of Eq. (23). In this, way we get a modified gravity action consistent with the HDE scenario.

\section{A. Acknowledgements}

The first author sincerely acknowledges the facilities provided to him by the Inter-University Centre for Astronomy and Astrophysics (IUCAA), Pune, India, during his visit in November, 2012 under the Visitor Associateship Programme.

[1] Perlmutter, S., et al., Astrophys. J., 517, 565 (1999).

[2] Bennett, C. L., et al., Astrophys. J., 583, 1 (2003).

[3] Spergel, D. N., et al., Astrophys. J. Suppl. Ser., 148, 175 (2003).

[4] Tegmark, M., et al., Phys. Rev. D, 69, 103501 (2004).

[5] Abazajian, K., et al., Astron. J., 128, 502 (2004).

[6] Abazajian, K., et al., Astron. J., 129, 1755 (2005).

[7] Allen, S. W., Schmidt, R. W., Ebeling, H., Fabian, A. C., and van Speybroeck, L., Mon. Not. R. Astron. Soc., 353, 457 (2004).

[8] Bennett, C. L., et al., Astrophys. J., 583, 1 (2003).

[9] Peebles, P. J. E., \& Ratra, B., Reviews of Modern Physics, 75, 559 (2003).

[10] Padmanabhan, T., Phys. Rep. 380, 235 (2003).

[11] Carroll, S. M. The New Cosmology: Conference on Strings and Cosmology, 743, 16 (2004).

[12] Bean, R., Carroll, S. and Trodden, M., 2005, arXiv:astro-ph/0510059.

[13] Carroll, S. M. Living Reviews in Relativity, 4, 1 (2001).

[14] Sahni, V. and Starobinsky, A., International Journal of Modern Physics D, 9, 373 (2000).

[15] Padmanabhan, T., Current Science, 88, 1057 (2005).

[16] Weinberg, S., Reviews of Modern Physics, 61, 1 (1989).

[17] Copeland, E. J., Sami, M. and Tsujikawa, S., International Journal of Modern Physics D, 15, 1753 (2006).

[18] Weinberg, S., Reviews of Modern Physics, 61, 1 (1989). 
[19] Wetterich, C., Nuclear Physics B, 302, 668 (1988).

[20] Ratra, B. and Peebles, P. J. E. Phys. Rev. D, 37, 3406 (1988).

[21] Zlatev, I., Wang, L. and Steinhardt, P. J., Physical Review Letters, 82, 896 (1999).

[22] Doran, M. and Jaumlckel, J., Phys. Rev. D, 66, 043519 (2002).

[23] Caldwell, R. R., Physics Letters B, 545, 23 (2002).

[24] Nojiri, S., and Odintsov, S. D., Physics Letters B, 562, 147 (2003).

[25] Jamil, M., Myrzakulov, Y., Razina, O. and Myrzakulov, R., Astrophys. Space Sci., 336, 315 (2011).

[26] Jamil, M., Momeni, D., Serikbayev, N. S. ande Myrzakulov, R., Astrophys. Space Sci., 339, 37 (2012).

[27] Armendariz-Picon, C., Mukhanov, V. and Steinhardt, P. J., Phys. Rev. Lett. 85, 4438 (2000).

[28] Armendariz-Picon, C., Mukhanov, V. and Steinhardt, P. J., Phys. Rev. D 63, 103510 (2001).

[29] Chiba, T., Okabe, T., and Yamaguchi, M., Phys. Rev. D 62, 023511 (2000).

[30] Fischler, W., Susskind, L., (arXiv:hep-th/9806039)

[31] 't Hooft, G. 1993, arXiv:gr-qc/9310026

[32] Cohen, A., Kaplan, D., Nelson, A., Phys. Rev. Lett. 82, 4971 (1999)

[33] Huang, Q.G., Li, M., J. Cosmol. Astropart. Phys. 8, 13 (2004).

[34] Jamil,M. et al., Int. J. Theor. Phys., 579 (2011).

[35] Hsu, S. D. H., Physics Letters B 594, 13 (2004).

[36] Jamil, M., Sheykhi, A., Farooq, M. U., International Journal of Modern Physics D 19, 1831 (2010).

[37] Jamil, M., Saridakis, E. N., Setare, M. R., Physics Letters B 679, 172 (2009).

[38] Jamil, M., Saridakis, E. N., J. Cosmol. Astropart. Phys. 7, 28 (2010).

[39] Jamil, M., Sheykhi, A., International Journal of Theoretical Physics 50, 625 (2011).

[40] Jamil, M., Farooq, M. U., International Journal of Theoretical Physics 49, 42 (2010).

[41] Jamil, M., Umar Farooq, M., J. Cosmol. Astropart. Phys. 3, 2 (2010).

[42] Jamil, M., Rashid, M. A., Astrophys. J. 56, 429 (2008).

[43] Jamil, M., Farooq, M. U., Rashid, M. A., European Physical Journal C 61, 471 (2009).

[44] Pasqua, A., Khodam-Mohammadi, A., Jamil, M., Myrzakulov, R., Astrophys. Space Sci., 340, 199 (2012).

[45] Pasqua, A., Jamil, M., Myrzakulov, R., Majeed, B., Phys. Scr., 86, 045004 (2012).

[46] Zhang, X. and Wu, F.-Q., Phys. Rev. D, 76, 023502 (2007).

[47] Zhang, X., \& Wu, F.-Q. Phys. Rev. D, 72, 043524 (2005).

[48] Li, M., Physics Letters B, 603, 1, (2004).

[49] Nojiri, S. and Odintsov, S. D. 2009, American Institute of Physics Conference Series, 1115, 212 (2009).

[50] Clifton, T., Ferreira, P. G., Padilla, A. and Skordis, C., Phys. Rep., 513, 1 (2012).

[51] Nojiri, S. and Odintsov, S. D., Phys. Rev. D, 74, 086005 (2006).

[52] Myrzakulov, R., Sáez-Gómez, D. and Tureanu, A., General Relativity and Gravitation, 43, 1671 (2011).

[53] Banijamali, A., Fazlpour, B. and Setare, M. R., Astrophys. Space Sci., 338, 327 (2012).

[54] Kiritsis, E. and Kofinas, G., Nuclear Physics B, 821, 467 (2009). 
[55] Nojiri, S. and Odintsov, S. D. 2005, Physics Letters B, 631, 1; Li, B., Barrow, J. D., \& Mota, D. F. 2007, Phys. Rev. D, 76, 044027

[56] Cai, Y.-F., Chen, S.-H., Dent, J. B., Dutta, S., and Saridakis, E. N., Classical and Quantum Gravity, 28, 215011 (2011).

[57] Ferraro, R., and Fiorini, F. 2007, Phys. Rev. D, 75, 084031.

[58] Li, B., Sotiriou, T. P. and Barrow, J. D., Phys. Rev. D, 83, 064035 (2011).

[59] Sotiriou, T. P., Li, B. and Barrow, J. D., Phys. Rev. D, 83, 104030 (2011).

[60] Bamba, K. and Geng, C.-Q., J. Cosmol. Astropart. Phys., 11, 8 (2011).

[61] Jamil, M., Yesmakhanova, K., Momeni, D. and Myrzakulov, R., Central European Journal of Physics, (2012)(Online first).

[62] Jamil, M., Momeni, D., Myrzakulov, R. and Rudra, P., J. Phys. Soc. Jpn. 18111 (2012).

[63] Myrzakulov, R., Eur. Phys. J. C 711752 (2011).

[64] Ferraro, R. and Fiorini, F., Phys. Rev. D 75084031 (2007).

[65] Setare, M. R., Int. J. Mod. Phys. D 122219 (2008).

[66] Bamba, K., Myrzakulov, R., Nojiri, S., \& Odintsov, S. D. Phys. Rev. D 85, 104036 (2012)

[67] Bamba, K., Capozziello, S., Nojiri, S., \& Odintsov, S. D. Astrophys. Space Sci., 342, 155 (2012)

[68] Rastkar, A. R., Setare, M. R. and Darabi, F., Astrophys. Space Sci. 337487 (2012). 